\title{
Towards Organizational Development In Digital Organizational Twin
}

https://doi.org/10.21272/sec.5(3).126-133.2021

Olena Skrynnyk, ORCID: https://orcid.org/0000-0001-8300-6616

Postgraduate Student, Technology manager, Modis, Stuttgart, Germany

\begin{abstract}
Sustained continuous monitoring and replication of organizational development in digital organizational twins is of particular importance for labour-intensive enterprises and also those in which reciprocal relations between social, corporate, normative and performative aspects assume the leading role.

The main purpose of the research is the developing of a digital representation of organizational processes, which focuses on the performance, working activities, organizational issues, behaviour and interactions between of the organizational members. Consequently, the objectives of research include the monitoring of current research state, concept and design of a digital twin. The implementation of digital organizational twin should improve considering timely optimization of proactive and reactive organizational development measures in the company in relation to the core variables of the 7S model. The created digital twin should map the dynamics of organizational development, as well as concomitant and deviating processes.

Systematization literary sources and approaches for the digital replication of organizational development issues indicates the lack of publications on research and diffuse distribution of scientific interest. The initial design of organizational development in the digital twin is based on four main objects and limited to a certain number of investigated parameters. This paper compare the conventional and digitalized organizational development process, explain the data flow in digital organizational twin, the design of organizational development in the digital organizational twin, provide an overview of the individual facets of organizational development, list the parameterization models and exemplarily illustrate the visualization of selected parameters.

The results of the research can be useful for the expansion of the tension bridge between organisational development and technologies and the development of new potentials for the study of socio-technical effects in companies. This can be extended to include the other facets of business management and supplemented by the connection of other technological resources.
\end{abstract}

Keywords: digital organizational twin, organizational development, organizational management, organizational change, artificial intelligence.

JEL Classification: L22, O39.

Cite as: Skrynnyk, O. (2021). Towards Organizational Development In Digital Organizational Twin. SocioEconomic Challenges, 5(3), 126-133. https://doi.org/10.21272/sec.5(3).126-133.2021.

Received: 21.06 .2021

Accepted: 03.09 .2021

Published: 13.09 .2021

Copyright: (C) 2021 by the author. Licensee Sumy State University, Ukraine. This article is an open access article distributed under the terms and conditions of the Creative Commons Attribution (CC BY) license (https://creativecommons.org/licenses/by/4.0/).

\section{Introduction}

Turbulent economic development requires better agility from organizations. This goal is achievable via various approaches. In addition to the positive effects of sustainability and innovation is technologisation by implementing advanced digital technologies (Bilan et al., 2019), (Bilan et al., 2020), (Khan, 2018), (Umadia and Kasztelnik 2020), (Vasilyeva et al., 2019), (Zakharkin et al., 2018). 
On the way to virtualize business processes, many companies replicate the digital models of real production. The term digital twin is not a buzzword anymore, but rather the term of the replication of the real object, process or system in order to optimize certain processes, identify failures and make forecasts. Such digital replications of products, production, storage and distribution centers, as well as supporting processes and services, have been successfully applied by some companies. Conversely, digital organizational twins have not yet been widely used, and the corresponding field of knowledge is currently being developed. The review of latest scientific publications and market analysis of existing software solutions in this area reveal that in scientific as well as in economic environments, it is increasingly tending to digital mapping of business processes and mutual relationships of its actuators. Considering the recent growth in the collection, analysis and optimization of social development of an organization, the general perspective is gaining in importance. This indicates the distinct replaced focus from the internal group and individual perspective to the global issues (Skrynnyk, 2019).

Conventional organizational diagnosis methods partly reflect the positive or negative organizational development, but they have certain disadvantages (subjectivity through distorted perception, over-/underestimation due to limited scope of tasks and influence, Halo effect, contrast error, rating of others within a narrow range, where they are rated in average, evaluation of individual situations or performances rather than of the whole) and are limited in their applicability to digital twins due to the time frame involved (no real-time monitoring). The other approach to gathering information about the state of the organization is to verify the relevant parameters over short periods of time.

Generally stated, the organization aims to achieve expected performance from its members while adhering to a predefined behavioural framework. The organization implements the conduct norms for its members. These are required for each member, often differing from the informal group norms. The group controls and directs the interactions of its members through the system of expected behavioural patterns. Each role requires the establishment of norms and sanctions in the groups. The group norms reflect the values of the company. From the point of view of mission, vision, goals and organizational culture, norms and values are unconditionally required for acceptance by all members of the organization.

Conventional organizational development focuses on the attitudes and behaviour of the organization's members and their social relationships in the context of norms, values, visions, mission, goals, etc. defined by the company. This approach centers on the participation of individuals or groups affected by change. Digital organizational twin offers added value for companies through productivity, performance, and predictability (Parmar et al., 2020). Furthermore, the digital twin provides a number of supporting and substitutive functions (Kostenko et al., 2018). And thereby enable operational functional extension for improved digital capturing of interdisciplinary processes. Hence, the potential benefits of collecting, analysing, optimizing and predicting organizational development at any given time prove to be highly significant.

\section{Current state}

The scientific topic of organizational digital twins is highly coveted and, according to the results of exploration, is currently being researched by scientists in many countries. The possibilities of representing (in)directly ascertainable parameters of the social systems in enterprises are examined despite the barriers of technical and technological systems and hurdles from the legislator.

Despite the fact that the well-known digital twins increasingly reproduce their data from the captured state changes of sensors, processes, etc., the reflection factors of social processes and state changes still remain to be clarified.

Some approaches for use of digital organizational twins are known hitherto: Kerremans considers particular suitability for replication of changes in business processes and digital transformation (Kerremans, 2018), Kaivooja et al. emphasizes integration with knowledge management (Kaivo-oja et al., 2019), Riss et. al. offers holistic approach to organizational mapping in the context of enterprise modeling (Riss et al., 2020), Dorrer describes the model of digital organizational maturity by replicating performance dynamics (Dorrer, 2020), Rumyantseva et. al. explore the mapping of employee motivation in digital twins through resource-based behavioural modelling (Rumyantseva et al., 2020). 
Every organization is unique, so there is no general template for organizational digital twin. However, digital twins have to fulfill special general requirements: harmonizing formats and protocols to ensure specific standards, providing data accessibility, the ability to monitor parameter changes (for example, by physical collectors), and providing a digital model with calibratable templates for each digital unit.

To replicate the internal organizational development process unter emphasizing of the multitude of aspects, digital organizational twin operate with an integrative approach comprised numerous models and corresponding algorithms.

\section{Digital organizational twin}

Considering the continuous changes, the concept of the digital twin, which has been known for several years, can be interpreted differently. Regardless of this fact the structure layers can be considered as four important ones: the data layer describing the physical object, the layer of data processing and storage, the layer of model, algorithm and analysis, and user interface layer.

Distinctions of a social system's digital twin vary on all four levels, starting with objects (e. g., emotions, decisions, group communication patterns, leadership degree, organizational processes, commitment index, value of cultural mechanisms, etc.). Due to the fact that such systems predominantly analyse, map and control the processes of interactions of system components, as well as their dynamic behaviour, the complexity of digital twins is significantly greater and ranges from versatile collection of data, combined models and algorithms and parallel sequential analyses.

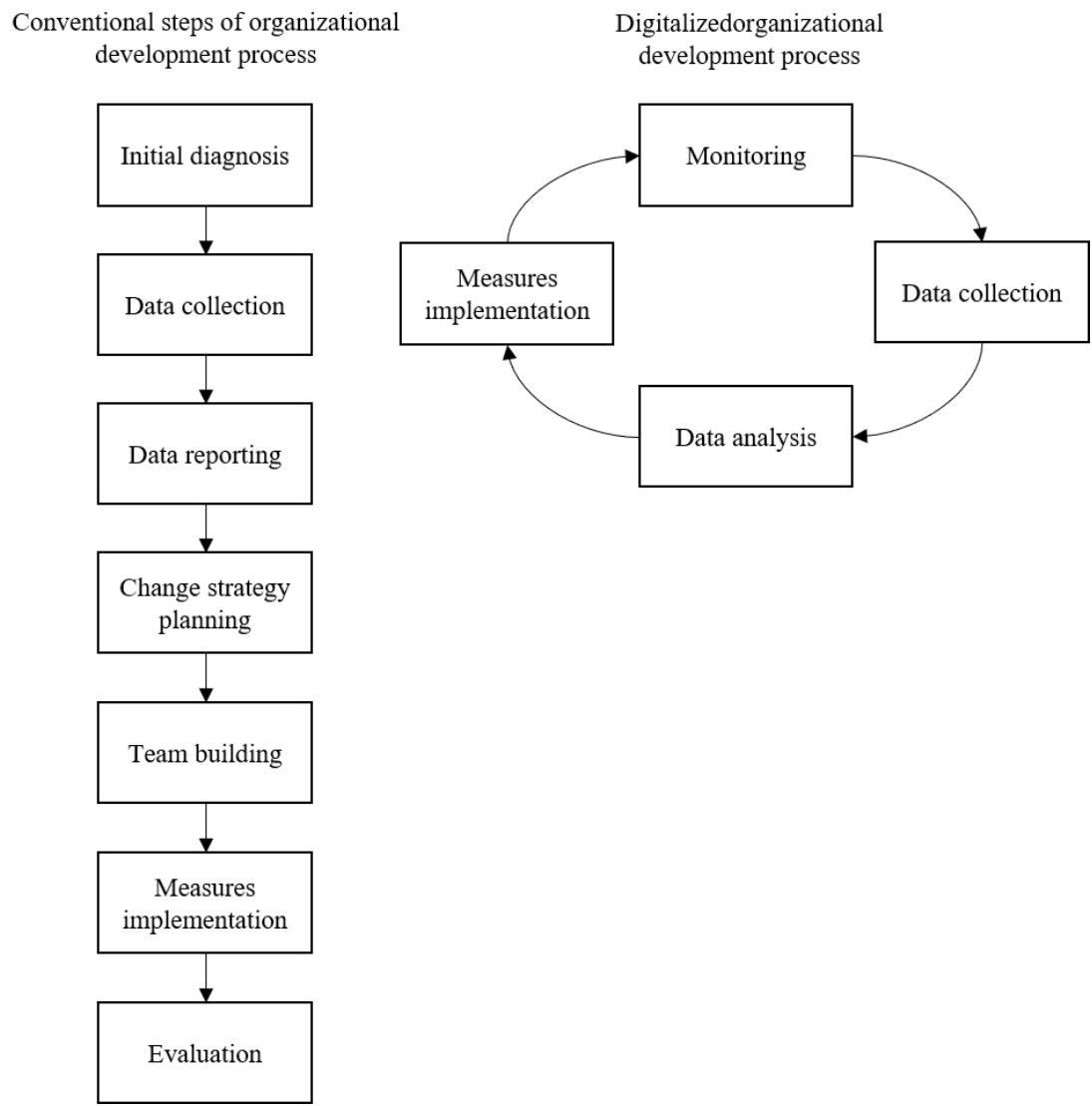

Figure 2. Comparison of conventional and digitalized organizational development process

Source: own processing. 
The collection of object related data, also in the context of organizational twin is a controversial issue due to different aspects: data protection, collection options, processing, and data storage capabilities. These issues pose a challenge for the development of a secure and protected digital twin.

The application of supporting or executive digital technologies for organizational development shifts the emphasis: the top-down strategy (initiation by management) is supplemented by cloud strategy (monitoring and control by software), changes at all levels are analysed and the insights are exploited for further transformation iterations. This elicits agreement between the process orientation of organizational development and the results' orientation of the IT system.

In order to successfully implement conventional change processes, certain measures should precede and accompany organizational development activities. These include initialization of organizational development/change activities, organizational diagnostics at all levels; learning, practising and improving competences, skills, knowledge; formation of special groups that guide and proactively support the transformation processes; intergroup interactions; measures to change the organizational and production structure to make processes and procedures more adaptable; consulting for management to support the assessment and improvement of internal organizational processes. In comparison, developed digital organizational twin is a tool that eliminates or largely supports some conventional measures. This has a stand-alone function in smaller organizations with manageable processes and dependencies between individual objects or a supporting function for already established processes in large organizations. The distinct advantages (continuous diagnostics, personalized adjustment of learning/training forms, learning effects of the system) are illustrated in Figure 1.

\section{Data flow in digital organizational twin}

Digital organizational twin provides automatic collection of data (e. g. on performance measurement, emotion and action recognition, KPIs, etc.), analysis of data, storage of analysis results and recommendation of actions based on the analysis results. Depending on the data type, four types of data processing and mapping are feasible (Figure 2).

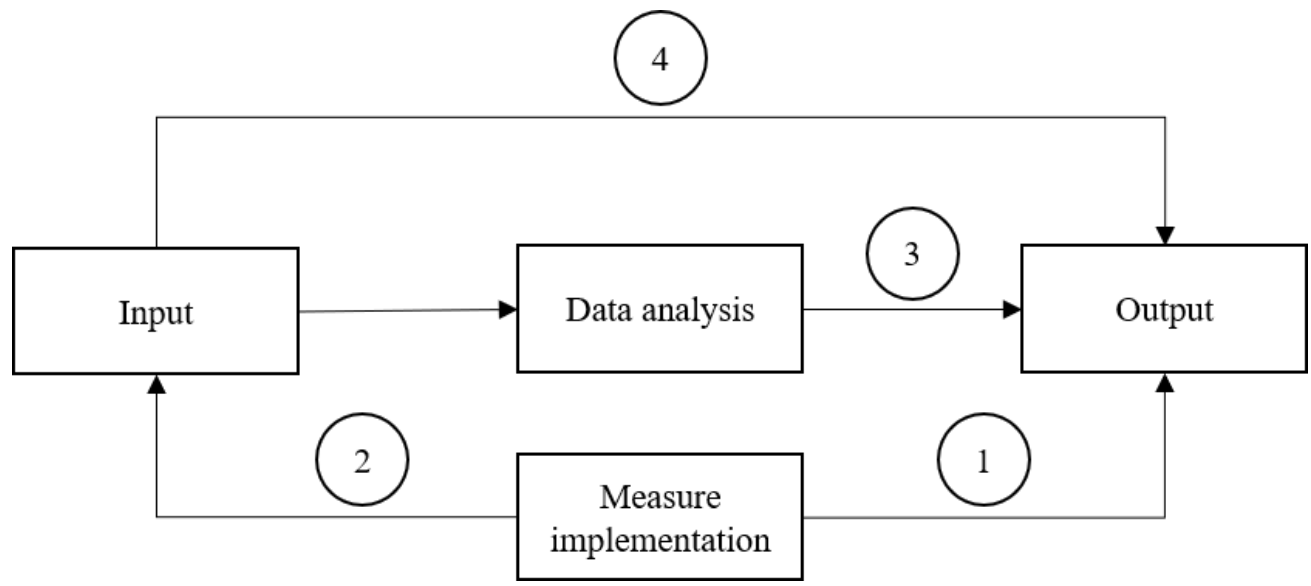

Figure 3. Data flow in digital organizational twin

Source: own processing.

According to the data flow, the parameter values are collected, analysed, corresponding measures are taken and issued (1) or their results are analysed again and further acted upon (2). The other data are only collected and analysed, the analysis results are output (3). Some parameter values are collected and displayed without additional analysis (4).

The collection of performance and decision-making data is performed via ERP systems or stand-alone or integrated devices, depending on the type of task. Information about the behaviour and activities of organisational members is gathered via stand-alone or integrated devices. Correspondingly, the data are processed and stored in private, public or hybrid cloud. 
Within the digital organizational twin, the replication of organizational aspects would be feasible in two respects: as a representation of the rigidly predefined key data (organizational development and change data e. g. mission, norms, standards, values, goals, position-/ job-/ role-related expected behavioural patterns, structure- and process characteristics etc.) and as a reflection of dynamically changing parameters (e. g. measurable current state, performance data, emotions and acts, decision effectiveness etc.).

\section{Key parameters, data models and analysis}

Initial digital representation contains information, content and impact models. Information model includes data artefacts (process flow and analytical models). Accordingly, content or context model comprises the expected behaviour of digital artefacts (e.g. status). Impact models, in contrast, represent the relationships between different digital artefacts. The template models for organizational development parameters are designed for organization, group and individual (employee and leader). These were selected according to scientific findings and literature analyses (Shvindina, 2017).

\section{Facets of organizational development}

The facets of organizational development represented by the digital twin comprise:

> Company: includes all cultural, structural, internal political and general behavioural parameters of the members.

$>$ Employees: covers performance, behavioural and motivational parameters.

$>$ Decision - decision-making parameters.

$>$ Work: is described by performance and work parameters.

Goals, strategies and mission statements as target measures and restrictions in the concrete models are not assigned to a separate facet.

\section{Parameterization models}

Previously, the parameters particularly at the individual level (employee) were assessed at certain intervals using various diagnostic methods, mainly assessments, appraisals etc. Some scientists consider it reasonable to collect the data from the information system of the company (Kerremans, 2018), the others plead for the extension of the source of reference (Riss et al., 2020). Digital organizational twin possessed contrasting approach to data collection. In addition to the measurable performance data, the digital twin also includes the KPI values and the analysed results of behavioural monitoring.

Concerning content, the parameters correlating in the models are arranged into the following groups: Performance parameters (organizational, group performance, performance differences, performance shares in total performance, time coefficients), organizational structure parameters (count of subordinate units, interdependencies), strategy parameters (commitment to strategy and mission, differentiation, flexibility/agility, continuity), culture and climate parameters (value-based unity, work climate, loyalty, satisfaction), leadership and management parameters (participation, delegation, decision-making), work-related parameters (work order, work execution pattern, collaboration), behavioural parameters (act, emotion, motivation, need, competence level and difference).

The models and algorithms of data, as well as corresponding analysis are made according to the studied object (employee, group, organization and as a separate unit - leader). Individual performance model, behaviour-needs model, adjustable competence model and leadership model are applied to the individual objects (employee, leader). Group performance model, group norms and standards models, culture-group model are implemented at the group level. Level of organization is described by interrelationships of organizational performance model, structural model, and culture-organization model. The models reciprocally reference each other. Some parameters of one model directly reflect the parameter of the other one (Bonamigo and Mendes, 2019), (Hadbaa and Boutti, 2019), (Tovmasyan and Minasyan, 2020), (Vidic, 2018). Furthermore, the digital rganizational twin applyes models for organizational diagnostics and the selection of appropriate measures. 
The parameters obtained in the facets reflect the current events in the organisation by tracking the state. Therefore, the organizational parameter models listed in Table 1 come into consideration. These parameters comprise only the characteristic determinants as an example.

Table 1. Parameter models forming the basis of the digital organisational twin

\begin{tabular}{|c|c|}
\hline Parameter model & Parameters \\
\hline \multicolumn{2}{|l|}{ Company facet } \\
\hline $\begin{array}{l}\text { Organizational culture } \\
\text { model }\end{array}$ & $\begin{array}{l}\text { organizational learning rate, cohesion (togetherness) degree, resilience grade, work climate, distraction } \\
\text { rate }\end{array}$ \\
\hline $\begin{array}{l}\text { Organizational structure } \\
\text { model }\end{array}$ & depth of leadership, number of leadership unis, span of control \\
\hline $\begin{array}{l}\text { Organizational strategy } \\
\text { model }\end{array}$ & implementation grade, achievement rate, resourcen differences* \\
\hline \multicolumn{2}{|l|}{ Employees facet } \\
\hline Motivation-need model & motivation optimum, degree of needs perception, strength of expression \\
\hline Emotion and act model & type of activity*, quality*, duration*, iteration* \\
\hline Leadership model & $\begin{array}{l}\text { degree of leadership, participation rate, delegation degree, effectiveness of motivation, effectiveness of } \\
\text { employees' work }\end{array}$ \\
\hline \multicolumn{2}{|l|}{ Decision facet } \\
\hline Decision-making model & effectiveness of decision making, input, output, duration* \\
\hline \multicolumn{2}{|l|}{ Work facet } \\
\hline Performance model & derformance, capability degree, achivement rate of performance, time coefficients, difference \\
\hline
\end{tabular}

Notes: * refers to several parameters or forms the combination of such parameters.

Linear and non-linear functions describe the model, optimization algorithms adjust individual functions and evolutionary algorithms predict the possible events. Depending on the content, the system processes Boolean or fuzzy data sets (Skrynnyk and Vasilyeva, 2020). Furthermore, for the simulation of dynamic processes and prediction of parameter or state changes, the deep and machine learning models described previously are implemented accordingly.

\section{Visualization of parameters}

Output of data performed visually in the form of diagrams (mapping of current and predicted state) or text on the screen (indication, recommendations, videos and corrective actions, chatbots), auditorily (signals, corrective actions, talkbots).

\section{Discussion}

Digitalization of organizational development has some limitations:

$>$ Digital twins are functional-oriented and in the case of organizational development, disregard some sociopsychological aspects.

$>$ The linking of link some design levels in the first step (organizational structure and organizational development) was deliberately omitted.

Due to privacy and information protection, this is mainly intended to reflect the dynamics of development and the changes in interactions and not the private data. Consequently, through deindividualisation of the objects under investigation the concentration on the organizational changes became feasible.

Due to holistic conception of digital twin developed by us, many object models are adaptable. These can be adjusted in further steps.

The significant advantages of digitalization of individual organizational development steps include the resource efficiency (costs, time), the potential for monitoring organizational processes independent of location, the 
selective implementation of appropriate measures ("customized" types of adjustments through the use of mobile technologies), and the continuous improvement of the system through the implementation of artificial intelligence.

Implementation of AR/VR for visual recording of system output forms the next stage of expansion.

The results of this study can be used by researchers for further elaboration and analysis, and by practitioners for implementation in digital twins of their organizations.

Funding: self-funded.

Author contribution: conceptualization, Olena Skrynnyk; data curation, Olena Skrynnyk; formal analysis, Olena Skrynnyk; funding acquisition, Olena Skrynnyk; investigation, Olena Skrynnyk; methodology, Olena Skrynnyk; project administration, Olena Skrynnyk; resources, Olena Skrynnyk; software, Olena Skrynnyk; supervision, Olena Skrynnyk; validation, Olena Skrynnyk; visualization, Olena Skrynnyk; writing - original draft, Olena Skrynnyk; writing - review \& editing, Olena Skrynnyk.

\section{References}

1. Bilan, Y., Brychko, M., Buriak, A., Vasilyeva, T. (2019). Financial, business and trust cycles: The issues of synchronization [Ciklusi financiranja, poslovanja i povjerenja: pitanja za sinkronizaciju]. Zbornik Radova Ekonomskog Fakultet au Rijeci, 37(1), 113-138. [Google Scholar], [CrossRef].

2. Bilan, Y., Pimonenko, T., Starchenko, L. (2020). Sustainable business models for innovation and success: Bibliometric analysis. E3S Web of Conferences, 159, 04037. [Google Scholar], [CrossRef].

3. Bonamigo, A., Mendes, D. (2019). Value Co-creation and Leadership: An Analysis Based on the Business Ecosystem Concept. Business Ethics and Leadership, 3(4), 66-73. [Google Scholar], [CrossRef].

4. Dorrer, M. V. (2020). The prototype of the organizational maturity model's digital twin of an educational institution. Journal of Physics: Conference Series 1691, 012121, 2-7. [Google Scholar], [CrossRef].

5. Hadbaa, H. \& Boutti, R. (2019). Behavioral Biases Influencing the Decision Making of Portfolio Managers of Capital Securities and Traders in Morocco. Financial Markets, Institutions and Risks, 3(1), 92-105. [Google Scholar], [CrossRef].

6. Kaivo-oja, J., Kuusi, O., Knudsen, M. S., Lauraeus, T. (2019). Digital twins approach and future knowledge management challenges: Where we shall need system integration, synergy analyses and synergy measurements? In: Communications in Computer and Information Science, 271-281. [Google Scholar], [CrossRef].

7. Khan, M. Y. H. (2018). The Effectiveness of Entrepreneurial Activities for Economic Development: A Route to Innovation and Job Generation. SocioEconomic Challenges, 2(2), 32-40, [Google Scholar], [CrossRef].

8. Kerremans, M. (2018). Market guide for process mining. Gartner Inc. [Link]. Accessed 15 March 2021.

9. Kostenko, D., Kudryashov, N., Maystrishin, M., Onufriev, V., Potekhin, V., Vasiliev, A. (2018). Digital Twin Applications: Diagnostics, Optimisation and Prediction. Proceedings of the 29th DAAAM International Symposium, 2018, 0574-0581. [Google Scholar], [CrossRef].

10. Parmar, R., Leiponen, A., Thomas, L. D. V. (2020). Building an organizational digital twin, Business Horizons, 63(6), 725-736. [Google Scholar], [CrossRef].

11. Riss, U. V., Maus, H., Javaid, S., Jilek, Ch. (2020). Digital Twins of an Organization for Enterprise Modeling. In: IFIP Working Conference on The Practice of Enterprise Modeling. PoEM 2020: The Practice of Enterprise Modeling, 25-40. [Google Scholar], [CrossRef].

12. Rumyantseva, I. A., Krotenko, T. Yu., Zhernakova, M. B. (2020). Assessment of employee motivation and demotivation as parameters of the "Digital twin of an organization's model". Vestnik Universiteta, 6, 186190. (In Russ.) [Google Scholar], [CrossRef].

13. Shvindina, H. (2017). Leadership as a driver for organizational change. Business Ethics and Leadership, 1(1), 74-82. [Google Scholar], [CrossRef].

14. Skrynnyk, O. (2019). Concept of artificial intellect for organizational development. Visnyk of Sumy State University, 4, 123-128, [Google Scholar], [CrossRef].

15. Skrynnyk, O., Vasilyeva, T. (2020). Neuro-Genetic Hybrid System for Management of Organizational Development Measures, CEUR, 2732, 20200411. [Link]. 
16. Tovmasyan, G., Minasyan, D. (2020). The Impact of Motivation on Work Efficiency for Both Employers and Employees also During COVID-19 Pandemic: Case Study from Armenia. Business Ethics and Leadership, 4(3), 25-35. [Google Scholar], [CrossRef].

17. Umadia, K. Sr., Kasztelnik, K. (2020). The Financial Innovative Business Strategies of Small to Medium Scale Enterprises in Developing Country and Influence for the Global Economy Performance, SocioEconomic Challenges, 4(3), 20-32. [Google Scholar], [CrossRef].

18. Vasilyeva, T., Kuzmenko, O., Bozhenko, V., Kolotilina, O. (2019). Assessment of the dynamics of bifurcation transformations in the economy. CEUR Workshop Proceedings 2422, 134-146. [Google Scholar], [CrossRef].

19. Vidic, F. (2018). Entrepreneurial Orientation and Knowledge Creation and Their Impact on Company Performance, SocioEconomic Challenges, 3(2), 37-48. [Google Scholar], [CrossRef].

20. Zakharkin, O. O., Basantsov, I. V., Myroshnychenko, I. O., Shcherbachenko, V. O. (2018). Analysis of the innovative development directions for industrial enterprises. Espacios, 40(27), 16-26. [Link]. 